\title{
The Magnetoresistance of Nanostructured Co-ZnO Films with ZnO Buffer-Layers
}

\author{
Xiaoli Li1,2, Long Cheng1, Yalei Wang1, Yan Gao1, Zhiyong Quan1, Xiufang Qin1, \\ Harry J. Blythe², Gillian A. Gehring ${ }^{2}$, Xiaohong Xu1 ${ }^{*}$ \\ ${ }^{1}$ Key Laboratory of Magnetic Molecules and Magnetic Information Materials of Ministry of Education, School \\ of Chemistry and Materials Science, Shanxi Normal University, Linfen, China \\ ${ }^{2}$ Department of Physics and Astronomy, University of Sheffield, Sheffield, UK \\ Email: ${ }^{*}$ xuxh@dns.sxnu.edu.cn
}

Received 3 September 2014; revised 3 October 2014; accepted 8 November 2014

Copyright (C) 2014 by authors and Scientific Research Publishing Inc.

This work is licensed under the Creative Commons Attribution International License (CC BY).

http://creativecommons.org/licenses/by/4.0/

(c) (i) Open Access

\begin{abstract}
Co-ZnO films were prepared on oxidised silicon by magnetron sputtering at room temperature both with and without a $\mathrm{ZnO}$ buffer-layer. The Co-ZnO films consisted of Co particles dispersed in a semiconductor matrix. The combination of a Co-ZnO layer and a ZnO buffer-layer has a higher magnetoresistance than the Co-ZnO layer alone on an insulating Si substrate. The causes of this effect were investigated using $X$-ray photoelectron spectroscopy, depth profiling using Auger electron spectroscopy and electrical resistance as well as measurements of the change in the saturation magnetisation, the field cooled- and zero field cooled-magnetisation. This work has shown clearly what criteria are needed to optimise the magnetoresistance and how these conditions may be met by adding a buffer-layer thus making granular films based on ZnO more suitable for applications as field sensors.
\end{abstract}

\section{Keywords}

Magnetron Sputtering, Magnetoresistance, ZnO Buffer-Layer

\section{Introduction}

The discovery of the giant magnetoresistive effect (GMR) [1] [2] is considered to have been the beginning of spintronics as an active research field [3]. Extensive studies of the magnetoresistance (MR) of heterogeneous structures have been performed with a view towards spintronic applications. MR is observed in layered magnetic structures composed of alternate ferromagnetic and non-magnetic layers [4]-[7] and can also be observed in

\footnotetext{
${ }^{*}$ Corresponding author.

How to cite this paper: Li, X.L., Cheng, L., Wang, Y.L., Gao, Y., Quan, Z.Y., Qin, X.F., Blythe, H.J., Gehring, G.A. and Xu, X.H. (2014) The Magnetoresistance of Nanostructured Co-ZnO Films with ZnO Buffer Layers. Materials Sciences and Applications, 5, 996-1003. http://dx.doi.org/10.4236/msa.2014.514101
} 
granular films, in which small ferromagnetic (FM) particles are dispersed in a non-magnetic matrix [8] [9]. The non-magnetic component (or matrix) may be either a metal or an insulator; GMR or tunnelling MR is produced accordingly. Although a high spin-injection efficiency from a ferromagnetic metal into a metal or an insulator has been achieved, spin-injection from a FM metal into a semiconductor is still a challenging task, due to their large conductivity mis-match [10].

Dilute magnetic semiconductors offer an effective way to produce spin-injection into a semiconductor, whereby transition metal (TM) ions are uniformly substituted as cations into the semiconductor host lattice. Homogeneous, dilute magnetic semiconductors may be formed, of which doped $\mathrm{ZnO}$ is a typical example. Moreover, $\mathrm{ZnO}$ barrier-based magnetic tunnel junctions [11]-[13] have previously been investigated, providing evidence of spin-injection into a semiconductor. On account of their potential application in magnetic sensors, MR readheads and MR random access memory, inhomogeneous $\mathrm{TM}-\mathrm{ZnO}$ semiconductor systems have been specifically designed to maximize granular MR [14]-[17]. Compared with the multilayer films, the magnetic granular films have some advantages, such as simple preparation, good thermal stability, and tunable grain size and structure after deposition; this is helpful to maximize the use of the MR [9]. Moreover, MR in granular films can work with a magnetic field applied in any direction, whereas GMR tends to work only for magnetic field in plane. Many reports show that an oxygen deficiency is necessary to obtain a high magnetization in doped oxide semiconductor-based films [18] [19], which is also present in granular oxide films. Co-ZnO granular systems have the highest MR at room temperature (RT) and hence it is productive to look for ways of further improving these samples [20].

Experimental parameters, including the thicknesses of $\mathrm{Co}$ and $\mathrm{ZnO}$ layers and also post-annealing process, have been optimised in order to obtain large MR values as previously reported [15] [21]. However, by our knowledge, so far there has been no report about the influence on the MR of a $\mathrm{ZnO}$ buffer-layer between a $\mathrm{Co}-\mathrm{ZnO}$ granular layer and the substrate. In this work, we investigate the influence of a $\mathrm{ZnO}$ buffer-layer on the MR of Co-ZnO samples deposited on thermally oxidised silicon.

\section{Experiments}

Co-ZnO films of varying thickness, with or without a buffer-layer of $\mathrm{ZnO}$, were prepared on thermally oxidised $\mathrm{Si}(100)$ by magnetron sputtering at RT. The thickness of the $\mathrm{ZnO}$ buffer-layers were chosen to be $5 \mathrm{~nm}, 10 \mathrm{~nm}$, $15 \mathrm{~nm}, 20 \mathrm{~nm}, 35 \mathrm{~nm}, 50 \mathrm{~nm}, 75 \mathrm{~nm}, 100 \mathrm{~nm}, 150 \mathrm{~nm}$. The nominal structure of Co-ZnO films in all the samples is $[\mathrm{Co}(0.6 \mathrm{~nm}) / \mathrm{ZnO}(0.7 \mathrm{~nm})]_{10}$; this was achieved by sequentially depositing an ultra-thin $0.6 \mathrm{~nm}$ Co layer and a $0.7 \mathrm{~nm} \mathrm{ZnO}$ layer for 10 periods. The Co and $\mathrm{ZnO}$ deposition rate is $0.041 \mathrm{~nm} / \mathrm{sec}$ and $0.056 \mathrm{~nm} / \mathrm{sec}$, respectively. For these thin layers, granular films (GFs) are formed, rather than a multilayer [16]. Therefore, for convenience, in this paper, we shall denote $[\mathrm{Co}(0.6 \mathrm{~nm}) / \mathrm{ZnO}(0.7 \mathrm{~nm})]_{10}$ as GF; all the samples reported here contain the same amount of Co.

The structures of the samples were investigated by X-ray diffraction, XRD and transmission electron microscopy, TEM. Auger electron spectroscopy, AES depth profiling was performed in order to obtain the composition of the samples and observe the diffusion at the interface between a GF layer and a buffer-layer or a substrate. X-ray photoelectron spectroscopy, XPS was also performed to investigate the composition and the chemical state of Co in the samples. The magnetic field dependence of MR at RT was measured by using a four-probe method with the current in the plane. The maximum applied magnetic field was $18 \mathrm{kOe}$. Zero-field-cooled and field-cooled (ZFC/FC) magnetic moments of the samples were measured from $2 \mathrm{~K}$ to $300 \mathrm{~K}$ in 100 Oe using a SQUID magnetometer with a field applied parallel to the film plane. The magnetic properties of the thin films were measured using SQUID magnetometer at $5 \mathrm{~K}$.

\section{Results and Discussion}

The observed MR for GF-based samples in the maximum applied magnetic field of $18 \mathrm{kOe}$ at RT is shown in Figure 1(a) and Figure 1 (b). The MR ratio is defined as $[R(H)-R(0)] / R(0) \times 100 \%$, where $R(H)$ and $R(0)$ are the resistances in an external magnetic field and zero field, respectively. The GF sample without a ZnO buffer-layer shows a negative MR ratio of $8.3 \%$ at RT and $18 \mathrm{kOe}$. Adding ZnO buffer-layers to the substrates before depositing the GF increases the MR ratios of GF-based samples. Initially, the MR of the samples increases strongly with the increase of $\mathrm{ZnO}$ buffer-layer thickness from $0 \mathrm{~nm}$ to $20 \mathrm{~nm}$, and thereafter it remains nearly constant when ZnO buffer-layer is increased from $20 \mathrm{~nm}$ to $150 \mathrm{~nm}$. The negative MR ratio rises to a maximum of $11.9 \%$ 
for $[\mathrm{Co}(0.6 \mathrm{~nm}) / \mathrm{ZnO}(0.7 \mathrm{~nm})]_{10}$ films with only 10 periods. It is also enhanced compared by about $11 \% \mathrm{MR}$ for the [Co $(0.6 \mathrm{~nm}) / \mathrm{ZnO}]_{60}$ samples with 60 periods as previously investigated [14] [15]. The origin of the higher MR ratio in the system combining a Co-ZnO layer plus a $\mathrm{ZnO}$ buffer-layer will be subsequently investigated in detail from microstructures, conductivity, and magnetic properties.

Figure 2(a) shows the XRD patterns of the GF samples with and without a $\mathrm{ZnO}(50 \mathrm{~nm})$ buffer-layer. The XRD pattern of GF sample without a ZnO buffer-layer does not show any peaks, which means that this sample is in an amorphous state or the crystal grains are too small to be detected by XRD [15]. The sample of the GF with a $\mathrm{ZnO}(50 \mathrm{~nm}$ ) buffer-layer gives rise to a barely-discernible broad $\mathrm{ZnO}$ (002) peak. This data shows that our samples have small grain size and poor crystallinity because of the low substrate temperature, RT, which can also be evidenced from the TEM micrograph as shown in Figure 2(b). No lattice fringes are clearly observed, which implies poor crystallinity of the sample. It can be seen that the sample consists of Co particles (dark regions) dispersed in a semiconducting matrix (light regions), which is similar to that found in our previous studies of $\mathrm{Co} / \mathrm{ZnAlO}$ films [17]. The interface between Co particles and semiconductor matrix is not distinct, suggesting that there is a concentration gradient of $\mathrm{Co}$ in the $\mathrm{ZnO}$. The gradient boundary layer between the $\mathrm{Co}$ and the $\mathrm{ZnO}$ was considered in detail previously [17].

The metallic Co fraction in the GF layer was obtained from XPS. The Co 2p core-level XPS spectrum of the GF sample with a $\mathrm{ZnO}(20 \mathrm{~nm})$ buffer-layer is shown in Figure 3(a). The peaks with the binding energy at 778.6 and $793.6 \mathrm{eV}$ originate from Co metal and the peaks at 780.3 and $795.6 \mathrm{eV}$ originate from the Co $2 \mathrm{p}_{3 / 2}$ and $\mathrm{Co} 2 \mathrm{p}_{1 / 2}$ of $\mathrm{Co}^{2+}$ ions. Their respective weak satellite peaks occur at higher binding energies. This means that Co in the sample exists as metallic $\mathrm{Co}$ and as $\mathrm{Co}^{2+}$ ions, as occurred in our $\mathrm{Co} / \mathrm{ZnAlO}$ samples [17]. The ratio of me-

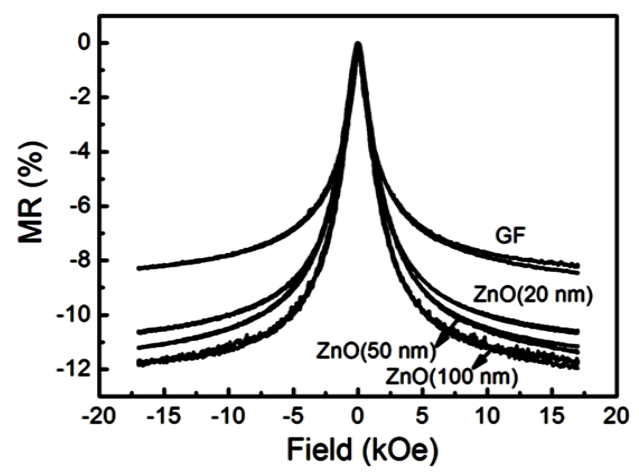

(a)

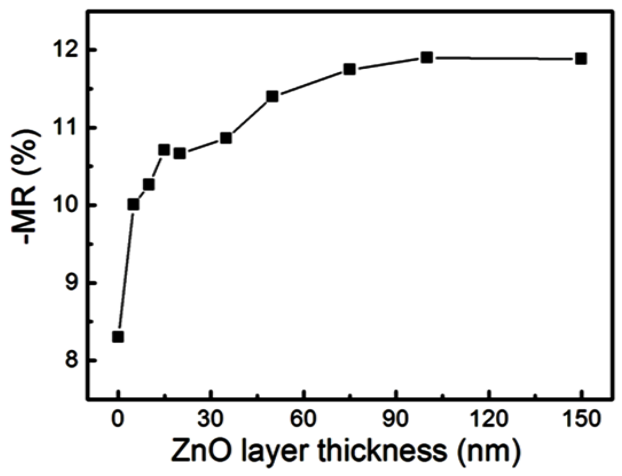

(b)

Figure 1. (a) The field dependence of MR ratios of GF-based samples at RT; (b) The dependence of the RT MR ratio in the maximum applied magnetic field of $18 \mathrm{kOe}$ for GF samples on a ZnO buffer-layer of thickness of 0 - $150 \mathrm{~nm}$.
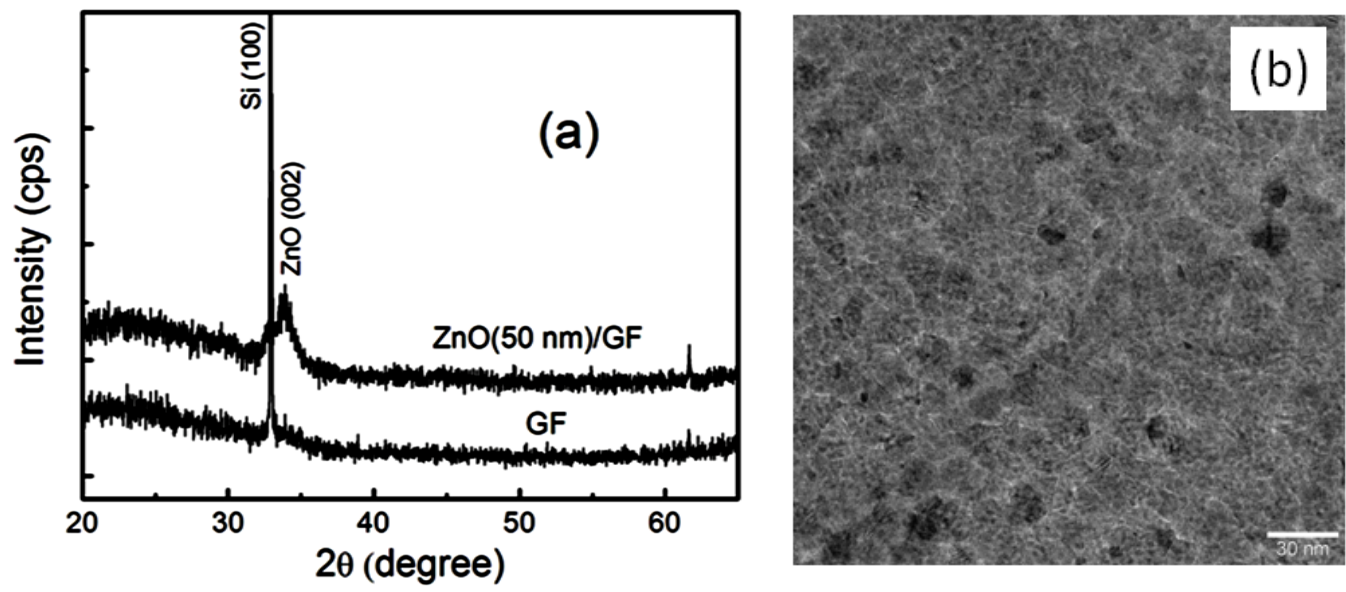

Figure 2. (a) XRD patterns of GFs without a buffer-layer and with a $\mathrm{ZnO}(50 \mathrm{~nm})$ buffer-layer; (b) TEM plane view image for the $\mathrm{ZnO}(50 \mathrm{~nm}) / \mathrm{GF}$ sample. 


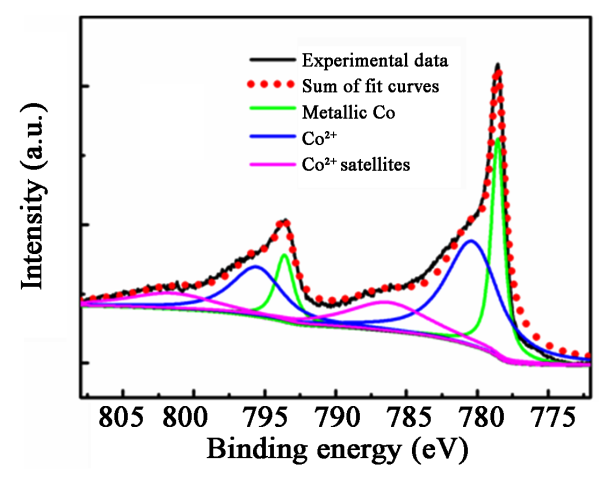

(a)

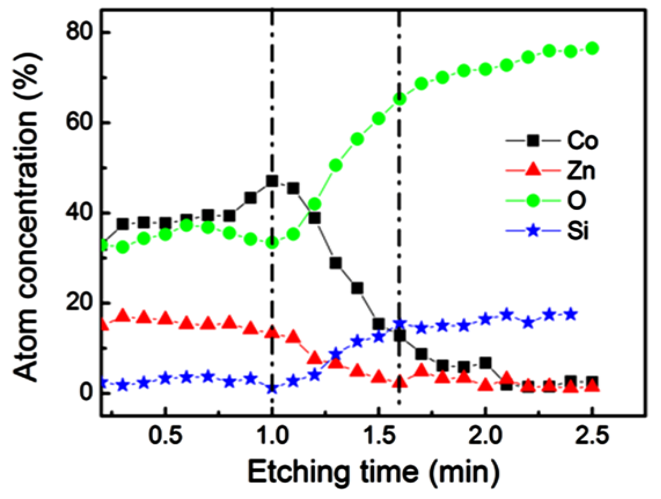

(b)

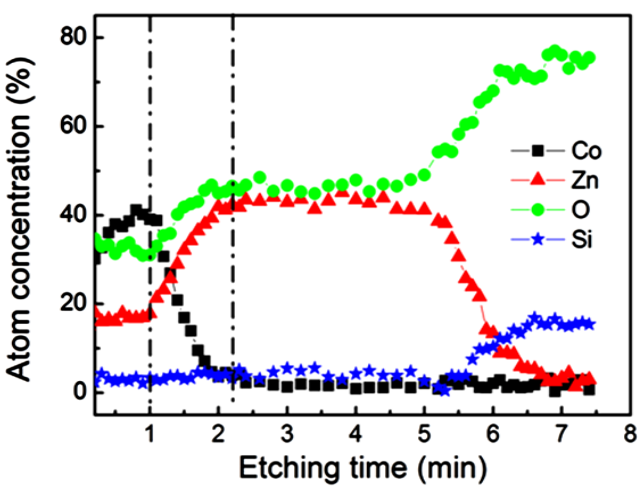

(c)

Figure 3. (a) Co 2p core-level XPS spectrum of the $\mathrm{ZnO}(20 \mathrm{~nm}) / \mathrm{GF}$ sample. It is fitted using the Gaussian method. The compositional depth profiles of GF samples without a buffer-layer (b) and with a $\mathrm{ZnO}(50 \mathrm{~nm})$ buffer-layer (c). The dash dot lines in Figure 3(b) and Figure 3(c) is a guide for the eye.

tallic Co to $\mathrm{Co}^{2+}$ ions for the GF layer is $\sim 0.5$ from a comparison between the areas of their $2 \mathrm{p}_{3 / 2}$ peaks, and the atom ratio of $\mathrm{Zn} / \mathrm{Co}$ of $\sim 0.6$ was also obtained from the XPS data. Therefore, the atomic concentration of metallic Co in the GF layer is equal to $1 /(1+0.6) \times 0.5 /(1+0.5)=20.8 \%$. This is considerably higher than that found in our previous study of $\mathrm{Co} / \mathrm{ZnAlO}$ samples where the metallic Co atomic concentration in that sample was only $4.09 \%$, resulting from a much lower ratio of metallic Co to $\mathrm{Co}^{2+}$ ions of $\sim 0.145$ [17]. The nominal structure of the film in this work is $[\mathrm{Co}(0.6 \mathrm{~nm}) / \mathrm{ZnO}(0.7 \mathrm{~nm})]_{10}$; the one in our previous work is $[\mathrm{Co}(0.6) / \mathrm{Al}-\mathrm{doped} \mathrm{ZnO}$ $(3.0 \mathrm{~nm})]_{60}$.

In order to analyse the interface between the GF layer and the substrate or the ZnO buffer-layer, AES compositional depth-profiles were obtained by $\mathrm{Ar}^{+}$etching. Results for the GF samples grown without a buffer-layer and with a ZnO (50 nm) buffer-layer are shown in Figure 3(b) and Figure 3(c), respectively. In the process of measuring AES, the standard etching $\mathrm{SiO}_{2}$ rate of $10 \mathrm{~nm} / \mathrm{min}$ is used as a reference for etching $\mathrm{GF}$ and $\mathrm{ZnO}(50$ $\mathrm{nm}$ /GF samples. It can be seen that the Co concentrations in the samples are initially almost independent of etching time from 0 to $\sim 1$ min. As shown in Figure 3(b) and Figure 3(c), the Co concentrations are decreased when the samples are etched further; this implies the beginning of etching of the interface of the GF/substrate and GF/ZnO layer. The deposited GF films should have a nominal thickness of $\sim 13 \mathrm{~nm}$ which corresponds to an etching time of $\sim 1.3$ minute in the AES. The gradual decrease of the Co signal at the interface is significantly longer for the film with the buffer-layer than for the GF on oxidised Si, $\sim 1.1$ min compared with $\sim 0.7$ min, which translate roughly into lengths of $\sim 11 \mathrm{~nm}$ and $\sim 7 \mathrm{~nm}$. This indicates that there is a more diffuse interface when the GF is grown on a buffer-layer possibly caused because the Co in the GF layer diffuses more easily into a $\mathrm{ZnO}$ buffer-layer than into the thermal oxidised Si substrates. Any diffusion of Co particles of the GF layer into the $\mathrm{ZnO}$ buffer-layer will act to dilute the Co particles in GF layer. Since the same nominal GF [Co(0.6 $\mathrm{nm}) / \mathrm{ZnO}(0.7 \mathrm{~nm})]_{10}$ was deposited, a thicker GF layer may be formed in the sample with a ZnO buffer-layer.

The composition of the GF layer was obtained by both AES and XPS. The concentrations of the three main elements $\mathrm{Zn}$, Co, and $\mathrm{O}$ were obtained by averaging the results from AES and XPS, in consideration of the cer- 
tain deviation from different methods. It is found that the concentrations of the three main elements Co, Zn, and $\mathrm{O}$ in GF layer are $39.3 \pm 2.0$ at.\%, $23.1 \pm 1.2$ at.\%, and $37.6 \pm 1.9$ at.\%, respectively; this also implies that the microstructure of the GF layer may be a mix of metallic Co particles and semiconducting $\mathrm{Zn}_{1-\mathrm{x}} \mathrm{Co}_{\mathrm{x}} \mathrm{O}_{1-\delta}$ grains. The above discussion of the XRD, TEM, XPS, and AES of the samples indicates that the GF layer has a granular structure, in which the Co particles are dispersed in a semiconductor matrix and that Co diffusion may occur at the interface between GF and ZnO buffer-layer.

Further information is also obtained from the dependence of the resistance on the thickness of the buffer-layer which is shown in Figure 4. The resistance of the GF is increased significantly by growing the GF on a thick buffer-layer. We note that if the buffer layer was providing a resistance in parallel with that of the GF, the measured resistance would have fallen. The rise in the measured resistance indicates that both the resistivity and the structure of the whole GF are affected by a buffer-layer that is significantly thicker than the roughness/diffusion length. The resistivity of the whole film depends on the size of the metallic nanoparticles, because of the Coulomb blockade, and also the resistivity of the semiconductor matrix $\mathrm{Zn}_{1-\mathrm{x}} \mathrm{Co}_{\mathrm{x}} \mathrm{O}_{1-\delta}$, which is dependent on the oxygen deficiency, $\delta$. Any migration of the Co atoms out of the GF and into the buffer-layer will reduce the oxygen deficiency in the GF and hence raise the overall resistance.

Figure 5(a) shows the hysteresis loops of the GF samples with the same measured area that were taken at $5 \mathrm{~K}$ after the background diamagnetic signal has been subtracted. This indicates that the presence of a buffer-layer has reduced the saturation magnetisation $\left(M_{\mathrm{s}}\right)$ by $\sim 20 \%$.

ZFC/FC magnetic moment measurements were also performed, in a field of 100 Oe, in order to study the magnetic behavior of the Co particles in the different GF-based samples, as shown in Figure 5(b). At low temperatures, a large bifurcation is observed between the ZFC and FC curves for the two samples, as is normally exhibited by superparamagnetic particles [22] [23]. The ZFC magnetizations show a maximum centered at 140 $\mathrm{K}$ for GF and $100 \mathrm{~K}$ for the GF plus a ZnO buffer-layer, which means that their blocking temperatures $\left(T_{b} \mathrm{~s}\right)$ are below RT. It can be clearly seen from Figure 5(b) that the width of the peak in the ZFC curve of the GF is larger for the film grown without a buffer-layer. This implies that the width of the size distribution of the Co nanoparticles has been reduced by the inclusion of the buffer layer. The variation in the size of the Co particles can also be observed from its TEM plane view image in Figure 2(b).

The difference between the Co particles in the GF grown with and without a buffer-layer is most pronounced in the Curie-Weiss plots made at temperatures well above the $T_{b} \mathrm{~s}$. The susceptibilities follow the Curie-Weiss relation, $\chi=C /(T+\theta)$, where $C_{\mathrm{GF}}=533.51 \mathrm{emu} \mathrm{Oe}{ }^{-1} \mathrm{deg}^{-1}, C_{\text {buffer } / \mathrm{GF}}=158.52 \mathrm{emu} \mathrm{Oe} \mathrm{deg}^{-1}, \theta_{\mathrm{GF}}=75.85 \mathrm{~K}$ and $\theta_{\text {buffer/GF }}=2.90 \mathrm{~K}$. A positive Curie-Weiss constant, $\theta$ value characterises the presence of an antiferromagnetic interaction between the nanoparticles. A good MR material has very little coupling between the magnetic clusters so that the magnetisation of each cluster is free to respond to an external field; hence the reduction in $\theta$ caused by the buffer-layer is very beneficial to an increased MR. This can be evidenced from the larger MR of GF with a buffer-layer compared with that of the GF without a buffer-layer.

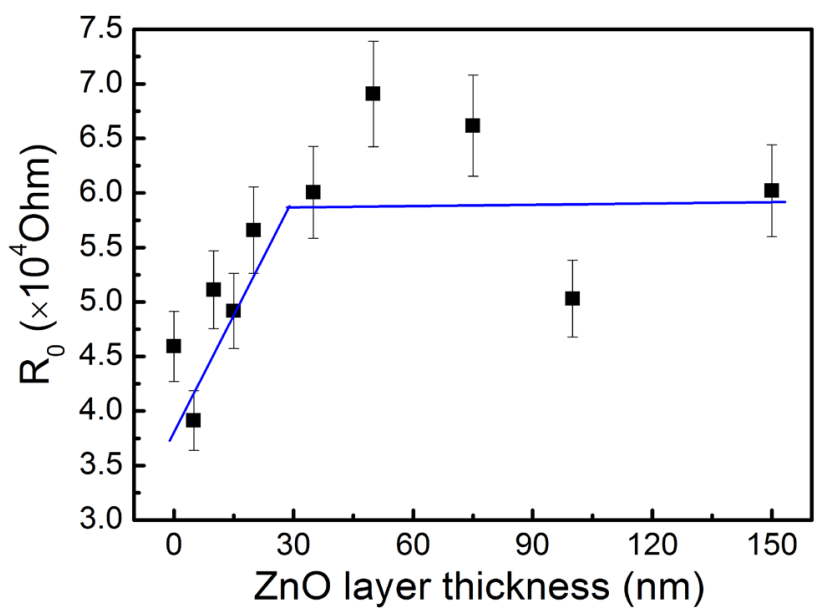

Figure 4. The dependence of the resistances for GF samples on a $\mathrm{ZnO}$ a buffer-layer of thickness of 0 - $150 \mathrm{~nm}$. The solid line is a guide for the eye. 


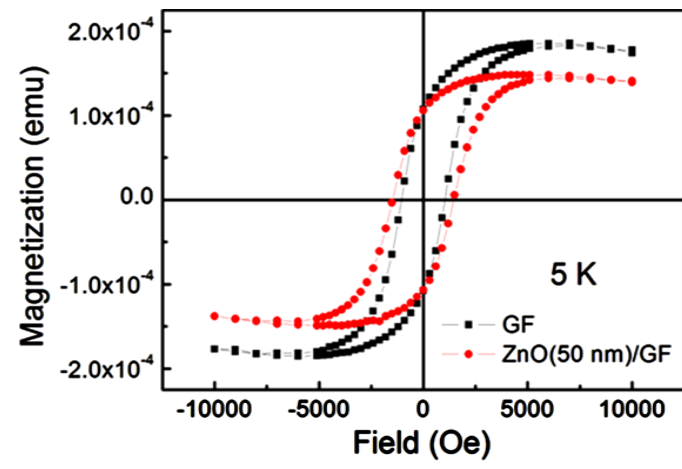

(A)

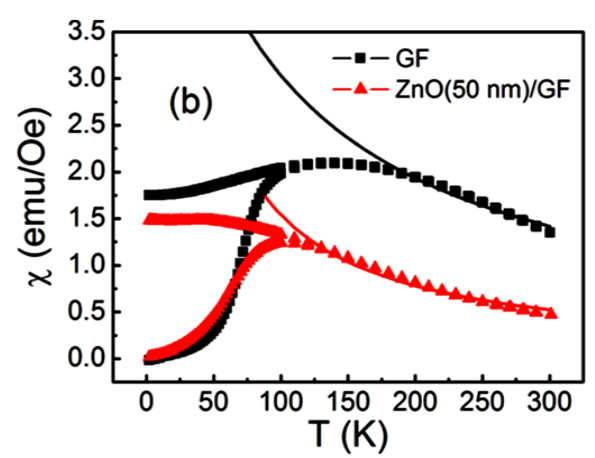

(B)

Figure 5. Magnetic data for GF's with and without a buffer-layer $(50 \mathrm{~nm}$ ). (a) The hysteresis loops obtained at $5 \mathrm{~K}$ after the diamagnetic background has been subtracted; (b) The temperature dependence of the susceptibilities found from the FC and ZFC magnetizations taken with a magnetic field of 100 Oe, the solid curves are fit to a Curie-Weiss law.

The change in the Curie constant, $C$, by a factor of $\sim 3$ indicates that the mean size of the nanoparticles has been reduced strongly by the inclusion of a $\mathrm{ZnO}$ buffer layer. The $T_{b}$ and the $M_{s}$ were smaller in the film grown with a buffer layer by $40 \%$ and $20 \%$. This indicates that the inclusion of the buffer layer has resulted in a modest decrease in the mean size of the nanoparticles but a much larger decrease in the average of the mean square size due to a reduction in the width of the size distribution due to the elimination of some of the largest nanoparticles.

According to the above discussion about microstructures and magnetic properties of the GF samples, there are two effects that act together to increase the MR in the films grown with a buffer layer. These are the reduction of the $\theta$ from $75.85 \mathrm{~K}$ to $2.9 \mathrm{~K}$ and the reduction of the size of the nanoparticles which increases the efficiency of the Coulomb blockade [24].

We now consider the physical processes that may be taking place at the interfaces between the GF and the silicon substrates and with the buffer. We have found that the inclusion of the buffer layer has resulted in a composite structure in which the mean size of the nanoparticles, the width of the particle size distribution, the $M_{\mathrm{s}}$ and the $C$ and $\theta$ have all decreased and the MR and the resistance have increased. The inclusion of the buffer layer will relieve strain and also can allow for cobalt atoms to diffuse into the $\mathrm{ZnO}$ buffer and may facilitate oxygen atoms to diffuse into the GF layer eliminating some of the oxygen vacancies.

There is evidence for interdiffusion at the interface between the GF and the buffer from the AES data which monitors the width of the interface which increases to $\sim 11 \mathrm{~nm}$ for a thick buffer. The MR and the resistance depend strongly on the thickness of the buffer layer for thicknesses less than $\sim 20 \mathrm{~nm}$ which is the range over which diffusion is likely to occur. The diffusion of Co out of the GF will reduce both the size of the nanoclusters as well as the amount of $\mathrm{Co}^{2+}$ in the semiconductor. This will reduce the magnetisation of the film and the size of the clusters. The diffusion of oxygen into the GF will reduce the concentration of oxygen vacancies which will increase the resistance and decrease the magnetism [19] of the semiconducting film. The larger density of oxygen vacancies in the semiconducting fraction of the GF on silicon will increase the magnetic interactions and, in this case, give rise to the higher $\theta$ value. The strain between the silicon oxide and the GF may induce the formation of large cobalt nanoparticles which are removed after the introduction of the buffer layer. It is the elimination of these large nanoparticles by the buffer which is reducing the mean square particle size so strongly.

\section{Conclusions}

In this work, the influence of ZnO buffer-layer on microstructures and MR properties of Co-ZnO samples was studied. Co-ZnO GF layer consisted of Co particles dispersed in a semiconductor matrix. Moreover, the interface between the GF and the buffer-layer is more diffuse than the GF on oxidised $\mathrm{Si}$, which may be due to the diffusion of $\mathrm{Co}$ and $\mathrm{O}$ ions between the GF and the ZnO buffer-layer. The combination of GF and a buffer-layer has a higher RT MR than the GF on an insulating Si substrate. The GF sample without a ZnO buffer-layer shows negative MR ratio of $8.3 \%$ at RT. Adding $\mathrm{ZnO}$ buffer-layers increases the negative MR ratio of GF-based sample to $11.9 \%$. The similar maximum of their ZFC magnetizations implies the similar average size of the Co particles; the only difference is the wider distribution of Co particle size in Co-ZnO sample without a buffer-layer. 
Therefore, these phenomena may be related with the difference of Co intergrain interactions, the distribution and the sizes of the Co nanoparticles, the number of oxygen vacancies in GF layer and the thickness of GF layer due to the diffusion at the interface in the sample with and without a $\mathrm{ZnO}$ buffer layer.

This paper has shown the combination of GF and a $\mathrm{ZnO}$ buffer-layer has a higher MR than the GF on an insulating Si substrate and has shown why this occurs. ZnO-based GF with a ZnO buffer-layer may be more suitable for applications as field sensors.

\section{Acknowledgements}

This work was supported by the National High Technology Research and Development Program of China (863 Program, No. 2014AA032904), the National Science Foundation of China (Nos. 51025101,11274214, 61306109), and the Research Foundation for the Doctoral Program of Higher Education (No. 20101404120002).

\section{References}

[1] Baibich, M.N., Broto, J.M., Fert, A., Nguyen Van Dau, F., Petroff, F., Eitenne, P., Creuzet, G., Friederich, A. and Chazelas, J. (1988) Giant Magnetoresistance of (001)Fe/(001)Cr Magnetic Superlattices. Physical Review Letters, 61, 2472. http://dx.doi.org/10.1103/PhysRevLett.61.2472

[2] Binasch, G., Grünberg, P., Saurenbach, F. and Zinn, W. (1989) Enhanced Magnetoresistance in Layered Magnetic Structures with Antiferromagnetic Interlayer Exchange. Physical Review Letters B, 39, 4828. http://dx.doi.org/10.1103/PhysRevB.39.4828

[3] Prinz, G.A. (1998) Magnetoelectronics. Science, 282, 1660. http://dx.doi.org/10.1126/science.282.5394.1660

[4] Greig, D., Hall, M.J., Hammond, C., Hickey, B.J., Ho, H.P., Howson, M.A., Walker, M.J., Wiser, N. and Wright, D.G. (1992) The Giant Magnetoresistance Co/Cu Superlattices Grown by MBE. Journal of Magnetism and Magnetic Materials, 110, L239. http://dx.doi.org/10.1016/0304-8853(92)90206-4

[5] Plaskett, T.S. and McGuire, T.R. (1993) Magnetoresistance in (Co10 $\AA / \mathrm{Cu} 10 \AA)_{\mathrm{n}}$ Multilayer Films as n Increases. Journal of Applied Physics, 73, 6378. http://dx.doi.org/10.1063/1.352656

[6] Morrow, P., Tang, X.T., Parker, T.C., Shima, M. and Wang, G.C. (2008) Magnetoresistance of Oblique Angle Deposited Multilayered Co/Cu Nanocolumns Measured by a Scanning Tunnelling Microscope. Nanotechnology, 19, 065712. http://dx.doi.org/10.1088/0957-4484/19/6/065712

[7] Wang, S.G., Ward, R.C.C., Du, G.X., Han, X.F., Wang, C. and Kohn, A. (2008) Temperature Dependence of Giant Tunnel Magnetoresistance in Epitaxial Fe/MgO/Fe MTJs. Physical Review Letters B, 78, 180411(R). http://dx.doi.org/10.1103/PhysRevB.78.180411

[8] Chien, C.L., Xiao, John, Q. and Samuel Jiang, J. (1993) Giant Negative Magnetoresistance in Granular Ferromagnetic Systems. Journal of Applied Physics, 73, 5309. http://dx.doi.org/10.1063/1.353765

[9] Peng, D.L., Wang, J.B., Wang, L.S., Liu, X.L., Wang, Z.W. and Chen, Y.Z. (2013) Electron Transport Properties of Magnetic Granular Films. Science China-Physics Mechanics \& Astronomy, 56, 15. http://dx.doi.org/10.1007/s11433-012-4969-1

[10] Schmidt, G., Ferrand, D., Molenkamp, L.W., Filip, A.T. and van Wees, B.J. (2000) Fundamental Obstacle for Electrical Spin Injection from a Ferromagnetic Metal into a Diffusive Semiconductor. Physical Review Letters B, 62, R4790. http://dx.doi.org/10.1103/PhysRevB.62.R4790

[11] Song, C., Liu, X.J., Zeng, F. and Pan, F. (2007) Fully Epitaxial Junction and Its Tunnel Magnetoresistance. Applied Physics Letters, 91, Article ID: 042106. http://dx.doi.org/10.1063/1.2762297

[12] Pan, F., Song, C., Liu, X.J., Yang, Y.C. and Zeng, F. (2008) Ferromagnetism and Possible Application in Spintronics of Transition-Metal-Doped ZnO Films. Materials Science and Engineering: R: Reports, 62, 1-35. http://dx.doi.org/10.1016/j.mser.2008.04.002

[13] Li, Q., Shen, T.T., Dai, Z.K., Cao, Y.L., Yan, S.S., Kang, S.S., Dai, Y.Y., Chen, Y.X., Liu, G.L. and Mei, L.M. (2012) Spin Polarization of $\mathrm{Zn}_{1-x} \mathrm{Co}_{x} \mathrm{O}$ Probed by Magnetoresistance. Applied Physics Letters, 101, Article ID: 172405. http://dx.doi.org/10.1063/1.4764542

[14] Yan, S.S., Ren, C., Wang, X., Xin, Y., Zhou, Z.X., Mei, L.M., Ren, M.J., Chen, Y.X., Liu, Y.H. and Garmestani, H. (2004) Ferromagnetism and Magnetoresistance of Co-ZnO Inhomogeneous Magnetic Semiconductor. Applied Physics Letters, 84, 2376. http://dx.doi.org/10.1063/1.1690881

[15] Li, X.L., Quan, Z.Y., Xu, X.H., Wu, H.S. and Gehring, G.A. (2008) Magnetoresistance in Co/ZnO Films. IEEE Transactions on Magnetics, 44, 2684-2687. http://dx.doi.org/10.1109/TMAG.2008.2003238

[16] Quan, Z.Y., Xu, X.H., Li, X.L., Feng, Q. and Gehring, G.A. (2010) Investigation of Structure and Magnetoresistance in 
Co/ZnO Films. Journal of Applied Physics, 108, Article ID: 103912. http://dx.doi.org/10.1063/1.3511752

[17] Quan, Z.Y., Zhang, X.P., Liu, W., Li, X.L., Addison, K., Gehring, G.A. and Xu, X.H. (2013) Enhanced Room Tempreture Magnetoresistance and Spin Injection from Metallic Cobalt in Co/ZnO and Co/ZnAlO Films. ACS Applied Materials \& Interfaces, 5, 3607-3613. http://dx.doi.org/10.1021/am303276b

[18] Park, S.Y., Kim, P.J., Lee, Y.P., Shin, S.W., Kim, T.H., Kang, J.H. and Rhee, J.Y. (2007) Realization of Room-Temperature Ferromagnetism and of Improved Carrier Mobility in Mn-Doped ZnO Film by Oxygen Deficiency, Introduced by Hydrogen and Heat Treatments. Advanced Materials, 19, 3496-3500. http://dx.doi.org/10.1002/adma.200602144

[19] Qi, S.F., Jiang, F.X., Fan, J.P., Wu, H.S., Zhang, S.B., Gehring, G.A., Zhang, Z.Y. and Xu, X.H. (2011) Carrier-Mediated Nonlocal Ferromagnetic Coupling between Local Magnetic Polarons in Fe-Doped $\operatorname{In}_{2} \mathrm{O}_{3}$ and Co-Doped ZnO. Physical Review B, 84, Article ID: 205204. http://dx.doi.org/10.1103/PhysRevB.84.205204

[20] Quan, Z.Y., Liu, W., Li, X.L., Xu, X.H., Addison, K., Score, D.S., Gehring, G.A. (2011) Structural and Magnetotransport Properties in Co/Nonmagnetic Films. Materials Letters, 65, 2982-2984. http://dx.doi.org/10.1016/j.matlet.2011.06.027

[21] Chen, Y.X., Yan, S.S., Liu, G.L., Mei, L.M. and Ren, M.J. (2007) Variations from $\mathrm{Zn}_{1-x} \mathrm{Co}_{x} \mathrm{O}$ Magnetic Semiconductor to Co-ZnCoO Granular Composite. Chinese Physics Letters, 24, 214. http://dx.doi.org/10.1088/0256-307X/24/1/058

[22] Jedrecy, N., von Bardeleben, H.J. and Demaille, D. (2009) High-Temperature Ferromagnetism by Means of Oriented Nanocolumns: Co Clustering in (Zn,Co)O. Physical Review B, 80, Article ID: 205204. http://dx.doi.org/10.1103/PhysRevB.80.205204

[23] Potzger, K. and Zhou, S. (2009) Non-DMS Related Ferromagnetism in Transition Metal Doped Zinc Oxide. Physica Status Solidi B, 246, 1147-1167. http://dx.doi.org/10.1002/pssb.200844272

[24] Mitani, S., Takahashi, S., Takanashi, K., Yakushiji, K., Maekawa, S. and Fujimori, H. (1998) Enhanced Magnetoresistance in Insulating Granular Systems: Evidence for Higher-Order Tunneling. Physical Review Letters, 81, 2799-2802. http://dx.doi.org/10.1103/PhysRevLett.81.2799 
Scientific Research Publishing (SCIRP) is one of the largest Open Access journal publishers. It is currently publishing more than 200 open access, online, peer-reviewed journals covering a wide range of academic disciplines. SCIRP serves the worldwide academic communities and contributes to the progress and application of science with its publication.

Other selected journals from SCIRP are listed as below. Submit your manuscript to us via either submit@scirp.org or Online Submission Portal.
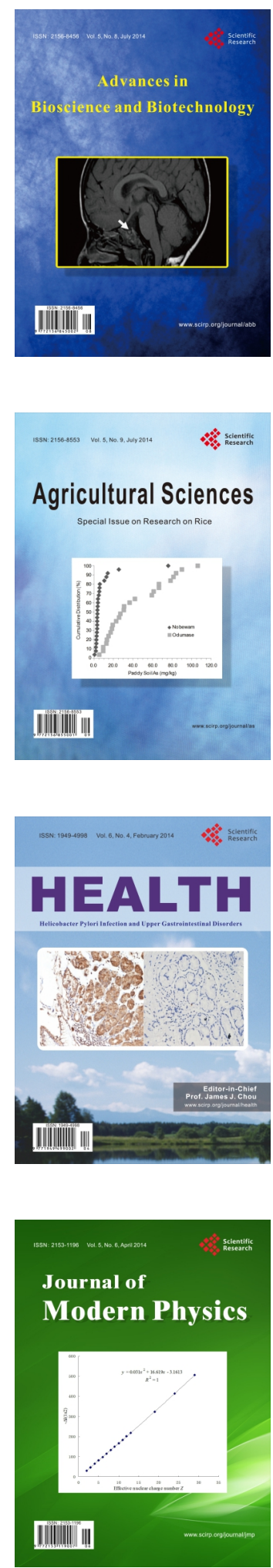
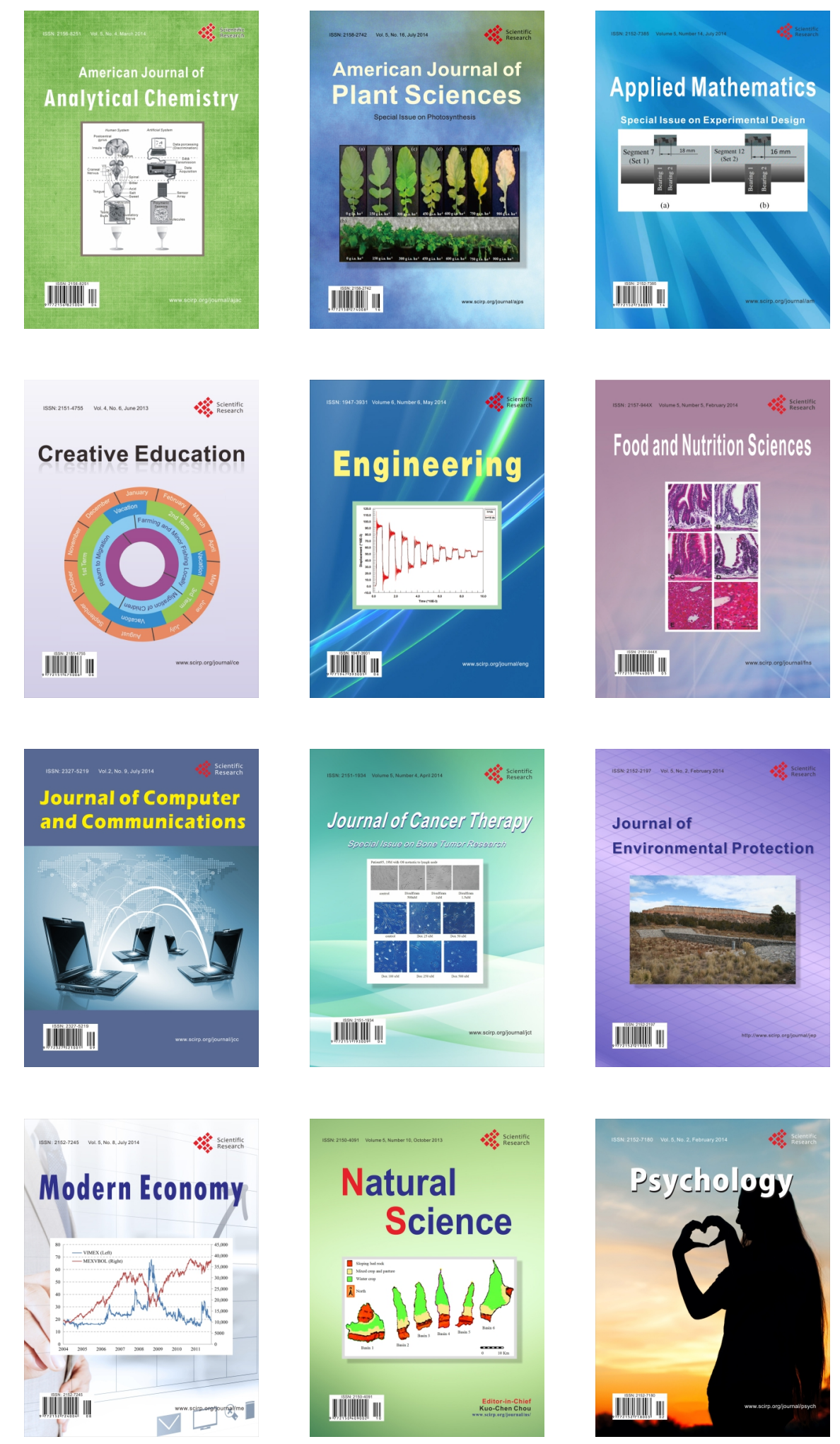